\title{
Topological vulnerability of the European power grid under errors and attacks
}

\author{
Martí Rosas-Casals ${ }^{1,2}$, Sergi Valverde ${ }^{2}$ and Ricard V. Solé S,3 $^{2,3}$ \\ ${ }^{1}$ Càtedra UNESCO de Sostenibilitat, Universitat Politècnica de Catalunya, EUETIT-Campus Terrassa, 08222 \\ Barcelona, Spain \\ ${ }^{2}$ ICREA-Complex Systems Lab., Universitat Pompeu Fabra, Dr. Aiguader 80, 08003 Barcelona, Spain \\ ${ }^{3}$ Santa Fe Institute, 1399 Hyde Park Road, New Mexico 87501, USA
}

\begin{abstract}
We present an analysis of the topological structure and static tolerance to errors and attacks of the September 2003 actualization of the Union for the Coordination of Transport of Electricity (UCTE) power grid, involving thirty-three different networks. Though every power grid studied has exponential degree distribution and most of them lack typical small-world topology, they display patterns of reaction to node loss similar to those observed in scale-free networks. We have found that the node removal behaviour can be logarithmically related to the power grid size. This logarithmic behaviour would suggest that, though size favours fragility, growth can reduce it. We conclude that, with the ever-growing demand for power and reliability, actual planning strategies to increase transmission systems would have to take into account this relative increase in vulnerability with size, in order facilitate and improve the power grid design and functioning.
\end{abstract}




\section{Introduction}

Mostly evolved over the last hundred and fifty years, technical infrastructures, from telegraph [Standage, 1998] to Internet [Pastor Satorras \& Vespignani, 2004], are the canvas where almost every aspect of our economy and society is portrayed. From a broader historical perspective, networks of energy, transportation and communication constitute the very foundation of all prospering societies, as the western culture actually knows them. Being usually managed by different kinds of actors (often with different objectives), formed by a huge quantity of heterogeneous components (spatially distributed and connected) characterized by complex interdependencies and relations, the study of these technological systems deserves attention in order to assure, essentially, structural integrity, efficiency and reliable supply.

In recent years, one particular kind of network has received much attention: the power grid. Hailed by the US National Academy of Engineers as the $20^{\text {th }}$ century's engineering innovation most beneficial to our civilization, the role of the electric power has grown steadily in both scope and importance during this time and electricity is recognized as a key to societal progress throughout the world, driving economy prosperity, security and improving the quality of life [Willis, 2004]. With similar pace, though, increasing frequency and size of malfunctions have raised general awareness about our real level of comprehension of these networks. In recent years, both the North American and the (once almost faultless) European grid systems have experienced numerous examples of such malfunctions in the form of cascading failures and blackouts [Venkatasubramanian, 2003; UCTE, September, 2003]. The explanations given by local, national and international electricity coordinating councils for most of these situations go from aspects related to low investment and maintenance, to those related to generation and demand inadequateness and, obviously, bad luck. But more than any, the most repeated explanation is that of a bad comprehension of the interdependencies present in the network. [Watts, 2003; UCTE, 2004]

In these sense, advances in statistical physics, modeling and computational methods have stimulated the interest of the scientific community to study electric power grids as complex networks. In complex network theory, one type of analysis of such interdependencies already mentioned is usually done under the robustness (or, in the contrary, vulnerability) epigraph [Boccaletti et al., 2006]. It refers to the ability of a network to avoid malfunctioning when a fraction of its constitutive elements is damaged. In technical infrastructures, this turns to be a field of elementary practical reasons since it affects directly the efficiency of the processes taking place in the network and it can give hints about the resilience of the grid. The analysis of the robustness of a complex system has been done, traditionally, from two points of view: static and dynamic. In a static robustness analysis, nodes are deleted without the need of redistributing any quantity transported by the network [Albert et al., 2000; Crucitti et al., 2003]. In a dynamic robustness analysis, nodes are deleted and the flow or load carried by them must be distributed over the rest of the remaining network [Moreno et al., 2002; Motter \& Lai, 2002; Crucitti et al., 2003; Kinney et al., 2005]. At first glance, the theoretical approach to these two types of robustness seems quite similar, but while static one can be analytically treated, dynamic one must be, almost always, numerically solved.

In this letter, the static robustness of the European and most of the European countries and regions electricity transport power grids are investigated. Their tolerance to random loss (failures) and selective removal (attacks) of the most connected nodes is analyzed. In order to simplify its topological representation, a simple model of the power grid data is introduced. Final results and some features worth to notice are discussed in the last part of the letter. 


\section{European power grid data}

In this paper, the vulnerability of the September 2003 actualization of the Union for the Coordination of Transmission of Electricity (UCTE) map has been analyzed. ${ }^{1}$ UCTE associates most of the continental Europe national power grid operators in order to coordinate the production and demand of some annual 2,300 TWh and 450 million customers from 24 countries. The map gives data from the transmission network (voltage levels from $110 \mathrm{kV}$ to $400 \mathrm{kV}$ ) and ignores the much more extended distribution one. Nonetheless, it deals with more than 3,000 nodes (generators and substations) and some $200.000 \mathrm{~km}$ of transmission lines.

For more than fifty years UCTE has coordinated the international operation of high-voltage European countries' grids to ensure adequate balances between offer and demand through national frontiers. It operates one of the largest electric synchronous interconnections worldwide in order to optimize the use of installed capacities and reduce the economic cost of power outages. But more than this, the UCTE transmission network has been shaped by those national policies and decisions that, for the last one hundred years, have been seeking economic prosperity, security and quality of life of its inhabitants. From that point of view, and differently from previous examples considered in the literature [Watts, 1999; Albert et al., 2004], those different power grids should be a good example of network evolution directed, at the same time, by technical, economical, political and, lastly, environmental decisions. Differentiated from country to country, we then would expect to find somehow different patterns and complex behaviour for every country or territory considered.

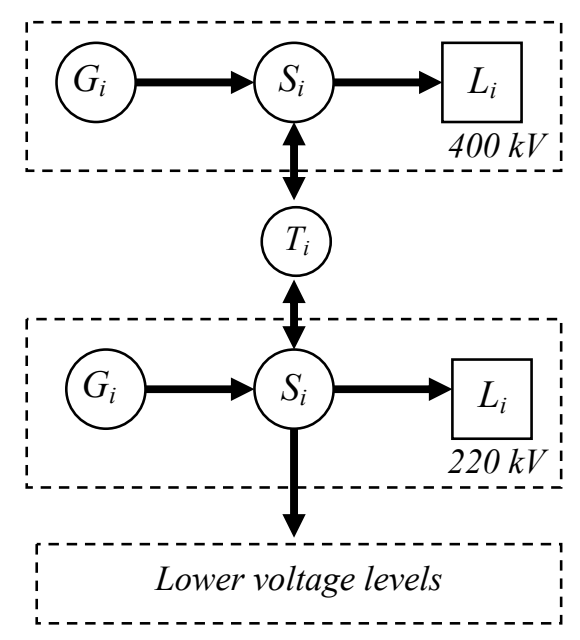

Fig. 1 An extremely simplified model for the transmission power grid: two voltage levels (400 kV and $220 \mathrm{kV}$ for the European network) with generators $G$ and loads $L$ connected by switching stations S. Transformers T connect both tension levels in order to provide reliability, efficiency and control capacity.

In order to simplify the analysis of the structure of the European power grid, an idealized view has been adopted (Fig. 1). On one hand, transmission lines have been assumed bidirectional, as it should be in the electricity transport network, and identical, ignoring the voltage level variation between lines and other physical characteristics. Although we have different voltage levels, the transport network works as a whole, using transformers to increase or decrease voltage depending on time and space requirements, and it would not be suitable or realistic to split it into different voltage networks, as it has been done in some literature [Crucitti et al., 2005]. On the other hand, although it is possible to distinguish four different kinds of elements, namely generators, transformers, switches (considered as stations or substations of any kind) and, finally, end line points, all these elements have been treated identically in order to avoid, at this initial point of the study, those difficulties involved in their differentiation and dynamical behaviour characterization.

\footnotetext{
${ }^{1}$ http://www.ucte.org
} 

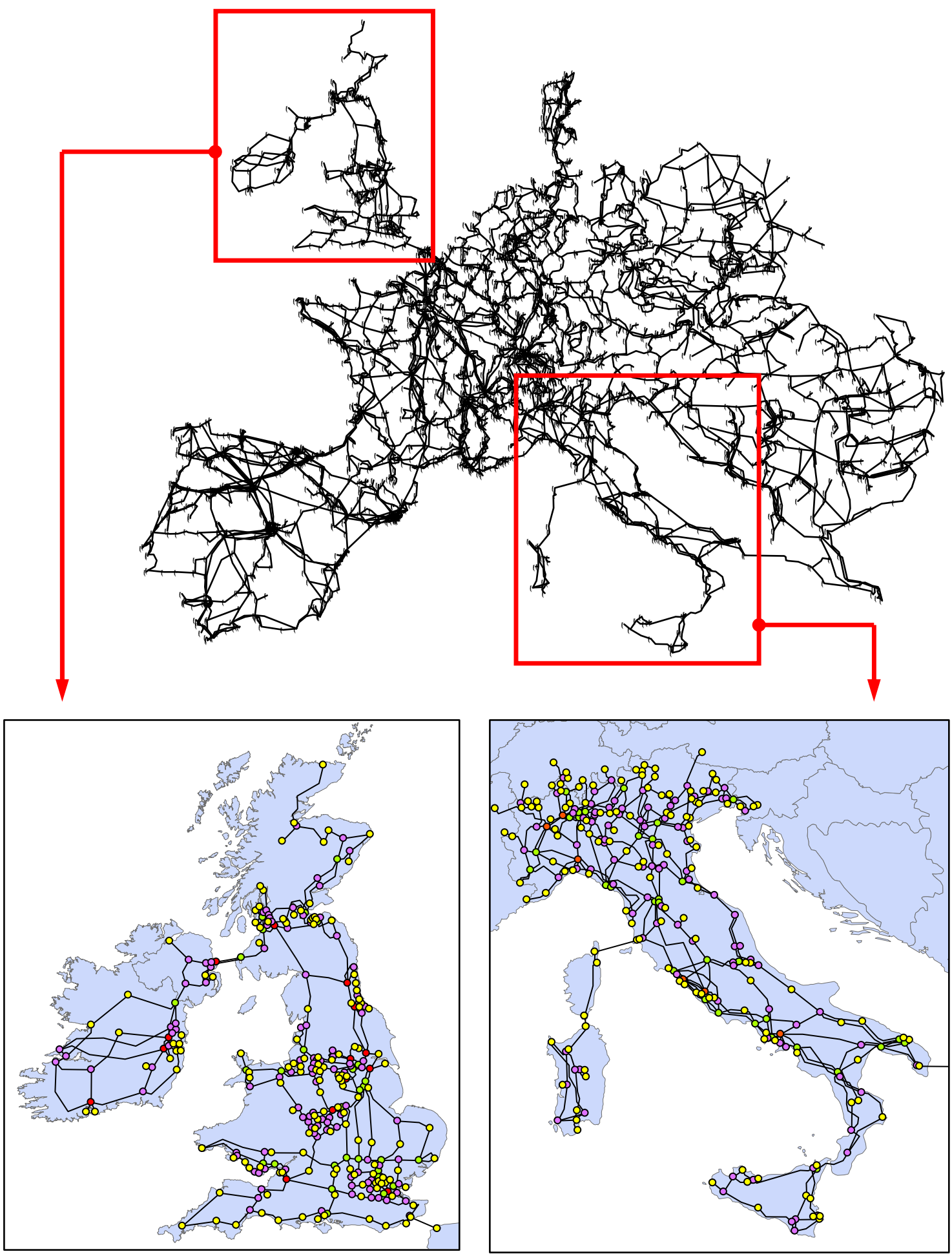

Fig. 2 The European electricity transport network (September 2003 actualization of the Union for the Co-ordination of Transmission of Electricity, UCTE, map) offers the upper topological image, where more than 3,000 nodes act as stations, substations, transformers and generators, connected by some 200,000 km of high voltage lines (up to 4,300 edges approximately). A closer look gives a more accurate perspective of some national power grids: United Kingdom and Ireland, bottom left, and Italy, bottom right. Size, and colour as well, indicate the degree of every node: 1 to 2 links, yellow; 3 to 4 links, purple; 5 to 6 links, green; and 7 to 8 links, red. 
Bearing these assumptions in mind, five different data sets have been analyzed:

- UCTE as a whole.

- UCTE, United Kingdom and Ireland as a whole.

- UCTE, country by country, plus United Kingdom and Ireland.

- Geographically related regions (Iberian Peninsula, Ireland as an island and England as an island).

- Traditionally united or separated regions (formers Yugoslavia, Czechoslovakia and Federal and Democratic Republics of Germany).

Until this time, and as far as we know, no such analysis has been done for the European power grid and with such depth of detail. A thorough analysis of these data sets will surely give hints of historical and geographical constraints that might have shaped the structure of the power grid from country to country, and from time to time. For example, from a geographical point of view, although neither United Kingdom nor Ireland belong to the UCTE, their isolated geography might have strongly configured and constrained their national power grids. Similarly, although Germany is actually united, the former frontier between Federal and Democratic Republics is still "visible" in form of a very few transmission lines connecting the east and the west of Germany.

\section{Small-world feature of the power grid}

The different data sets have been obtained after introducing their topological values, i.e. geographical positions of stations, substations and longitudes of lines, in a geographical information system (GIS) (Fig. 2). The national power grid for every country has been obtained from a typical GIS query: the selection of the part of the UCTE's network constrained by every country's frontier. So far, data analyses of 33 different networks have been performed.

Using the formalism of graph theory, any of these networks can be described in terms of an graph $\Omega$, defined as a pair, $\Omega=(W, E)$, where $W=\left\{w_{i}\right\},(i=1, \ldots, N)$ is the set of $N$ nodes and $E=\left\{w_{i}, w_{j}\right\}$ is the set of edges or connections between nodes. Here, $\xi_{i, j}=\left\{w_{i}, w_{j}\right\}$ indicates that there is an edge (and thus a link) between nodes $w_{i}$ and $w_{j}$. Two connected nodes are called adjacent, and the degree $k$ of a given node is the number of edges connecting it with other nodes. In this case, the UCTE graph, $\Omega_{U C T E}$, is defined as

$$
\Omega_{U C T E}=\bigcup_{i=1}^{n} \Omega_{i}
$$

where $\Omega_{i}(i=1, \ldots, n)$ are the set of national power grids analyzed.

As well as $k$, an additional property to be considered is the degree distribution $P(k)$. This is defined as the (normalized) probability that a node chosen uniformly at random has a degree $k$ or, similarly, as the fraction of nodes in the graph having $k$ edges. In this sense, it has been suggested that degree distributions can be classified in three types, namely exponential (gaussian or random), potential (scale-free) or some mixture of both, exhibiting each one different dynamic characteristics and adaptive behaviours [Amaral et al., 2000]. Most of the real networks degree distributions follow a power law of the form $P(k) \approx k^{-\gamma}$ with the exponent $\gamma$ being, mostly, between 2 and 3 .

For the five different data sets presented in Section 2, the graph model used considers undirected and unweighted edges. Though every single network contains hundreds of stations, 
substations, transformers and thousands of km of energy transport lines, the results show a surprising unity in mean degree, very similar to those encountered in the literature [Watts, 1999; Albert et al., 2004] for networks of the same size. The relation between nodes and links is constant and goes around $\langle k\rangle \cong 2,8$ for every network analyzed (Fig. 3). As it has been shown in the literature [Gastner \& Newman, 2004], though a rigorous demonstration of planarity is still elusive, this result agrees with that of other so called planar graphs like the US interstate highway network [Gastner \& Newman, 2004], ant network of galleries [Buhl et al., 2004] and urban networks [Buhl et al., 2006]. This would suggests that, although every technical infrastructure has evolved and developed under different economical, political, historical and, luckily enough, environmental conditions and decisions, there should be some universal characteristics related, almost surely, to the spatial and technological constraints that rule the construction and evolution of such networks in order to give a so common value for $\langle k\rangle$.

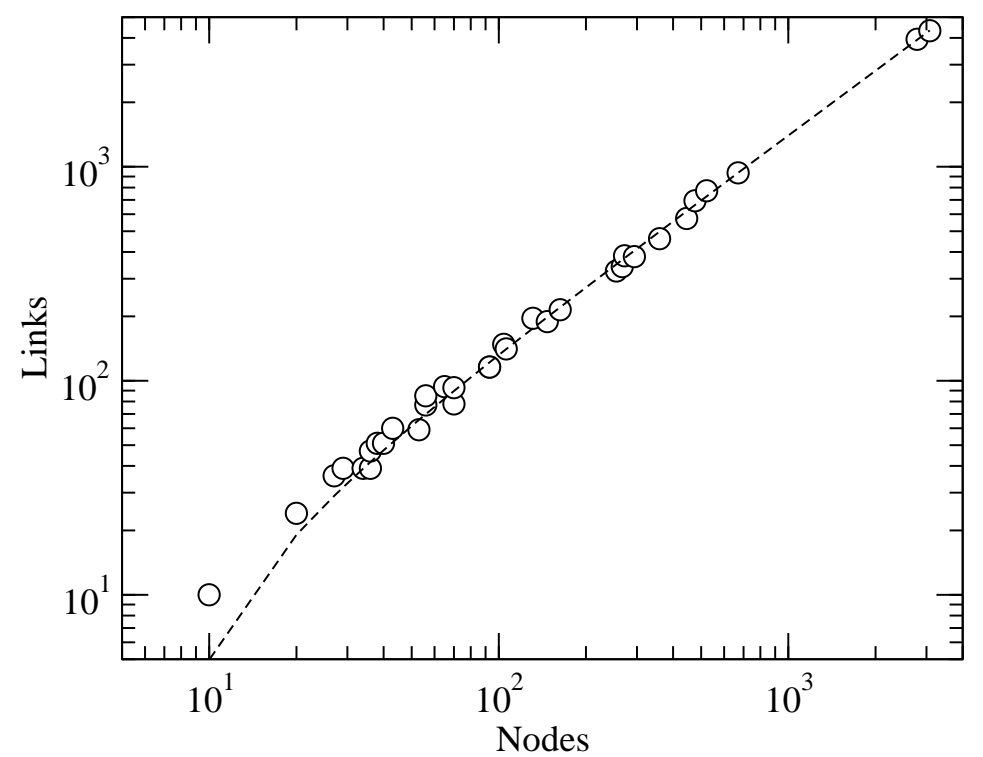

Fig. 3 Linear relation between nodes and links implies uniform mean degree value for every network analyzed, in spite of their different economical, political, historical and environmental evolution processes.

Every single network analyzed is uncorrelated (see below) and all of them follow an exponential cumulative probability degree distribution of the generic form

$$
P_{k^{\prime}>k}(k)=\int_{k^{\prime}}^{\infty} P(k) d k \approx \exp (-k / \gamma)
$$

The value adopted by the exponent $\gamma$ of these single scaled distributions goes from a minimum of $\gamma_{U K}=0.91$ (United Kingdom, with $\mathrm{r}^{2}=0.898$ ) to a maximum of $\gamma_{P T}=2.71$ (Portugal, with $\left.\mathrm{r}^{2}=0.989\right)$. The $\gamma$ exponent of the UCTE graph reaches a value of $\gamma_{U C T E}=1.78$, close to that of the North American power grid, as in Ref. [Albert et al., 2004]. The mean value for the whole data sets analyzed is $\bar{\gamma} \cong 1.8$ (Fig 4.a).

The presence of degree correlations (namely if connected nodes share common properties such as similar degrees) is conducted by measuring the average nearest neighbours connectivity of a node with degree $k$, i.e.

$$
\left\langle k_{n n}\right\rangle=\sum_{k^{\prime}} k^{\prime} P_{C}\left(k^{\prime} \mid k\right)
$$


where $P_{C}\left(k^{\prime} \mid k\right)$ is the conditional probability that a link belonging to a node with connectivity $k$ points to a node with connectivity $k^{\prime}$ [Pastor Satorras et al., 2001]. For we have independence on $k$, i.e.

$$
P_{C}\left(k^{\prime} \mid k\right)=P_{C}\left(k^{\prime}\right) \approx k^{\prime} P\left(k^{\prime}\right)
$$

and thus we have $\left\langle k_{n n}\right\rangle \approx$ constant (Fig. 4, b). Though no degree correlations has been found for the European power grid, for systems such as the Internet, such correlation exists and it is found that $\left\langle k_{n n}\right\rangle \approx k^{-v}$ with $v \cong 0,5$.
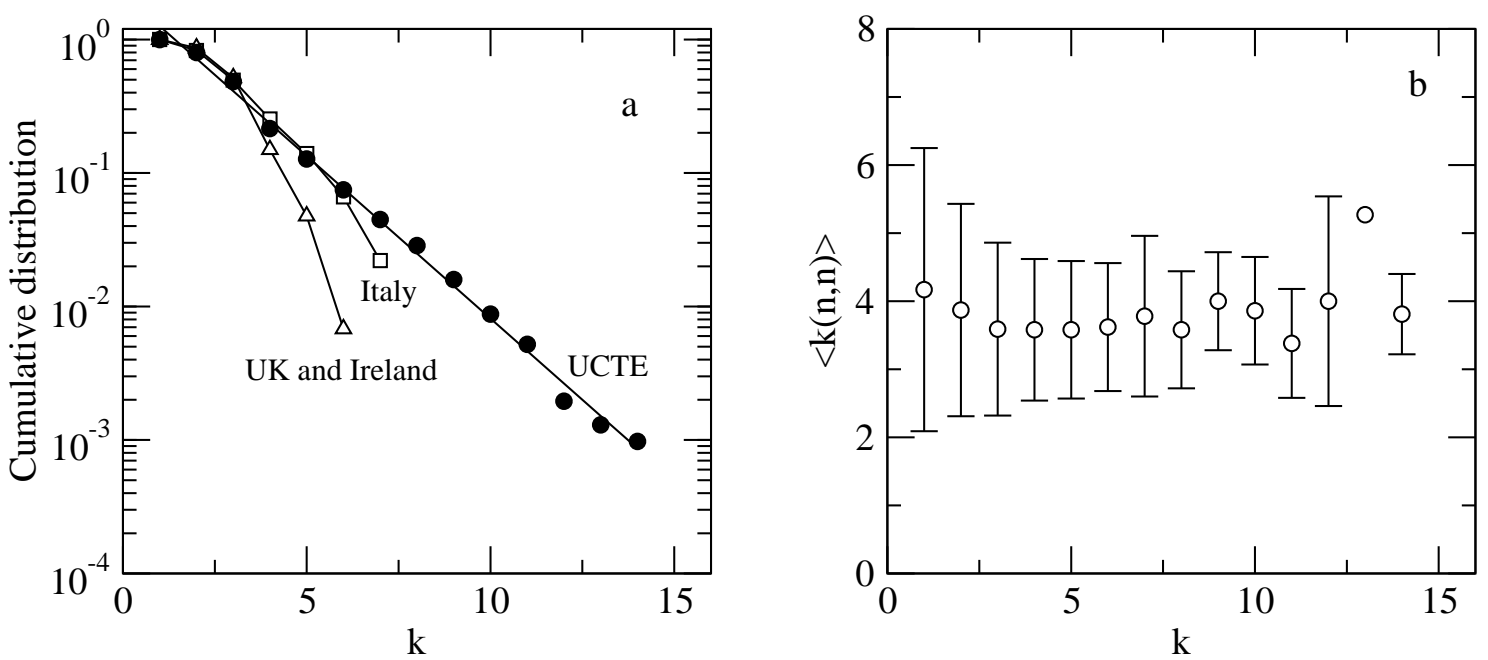

Fig. 4 Topological characteristics of the European power grid. (a) Cumulative degree distributions for the UCTE, (black dots, with exponential fitting), United Kingdom and Ireland (triangles) and Italy (squares) graphs. (b) Nearest neighbor degree correlation for the UCTE graph.

As well as the degree distribution, the small world (SW) feature has been used to characterize the topological structure of a network [Watts \& Strogatz, 1998]. The mathematical characterization of the SW behavior is based on the evaluation of two basic statistical properties: the clustering coefficient $C$, a measure of the average cliquishness of a node, and the characteristic path length $d$, a measure of the typical separation between two generic nodes in the network. On one hand, being $\Gamma_{i}=\left\{j \mid \xi_{i j}=1\right\}$ the set of nearest neighbors of a node $w_{i} \in W$, the clustering coefficient for this node is defined as the number of connections between the components $w_{j} \in \Gamma_{i}$. By defining

$$
\mathrm{Z}_{i}=\sum_{j=1}^{N} \xi_{i j}\left[\sum_{k \in \Gamma_{i} ; j<k} \xi_{j k}\right],
$$

we have $C_{v}(i)=Z_{i} /\left(\begin{array}{c}\left|\Gamma_{i}\right| \\ 2\end{array}\right)$, so that the clustering coefficient is the average over $W$,

$$
C=\frac{1}{N} \sum_{i=1}^{N} C_{v}(i)
$$


and measures the average fraction of pairs of neighbors of a node that are also neighbors of each other. On the other hand, being $d_{\text {min }}(i, j)$ the minimum path length connecting two nodes $w_{i}, w_{j} \in W$ in $\Omega$, we define the average path length of a given unit as

$$
d_{v}(i)=\frac{1}{N} \sum_{j=1}^{N} d_{\min }(i, j)
$$

and the path length for the graph as $d=\left\langle d_{v}(i)\right\rangle$. Characterized by small path lengths and high local clustering coefficient, the emergence of the SW phenomenon in some different real technological networks [Barabási \& Albert, 1999; Watts, 1999; Ferrer i Cancho et al., 2001; Albert et al., 2004] indicates that their connection topology is neither completely regular nor completely random: small-worlds are indeed highly clustered, like regular lattices, yet having small characteristic path lengths, like random graphs.

Here we use two predictions from random graph topologies in order to compare them against the observed topological patterns [Ferrer i Cancho et al., 2001]: (1) the clustering coefficient over the average connectivity for a random graph follows an inverse scaling law with graph size:

$$
\frac{C^{\text {rand }}}{\langle k\rangle}=\frac{1}{N}
$$

and (2), the average path length scales logarithmically as

$$
d^{\text {rand }} \log \langle k\rangle \approx \log (N)
$$
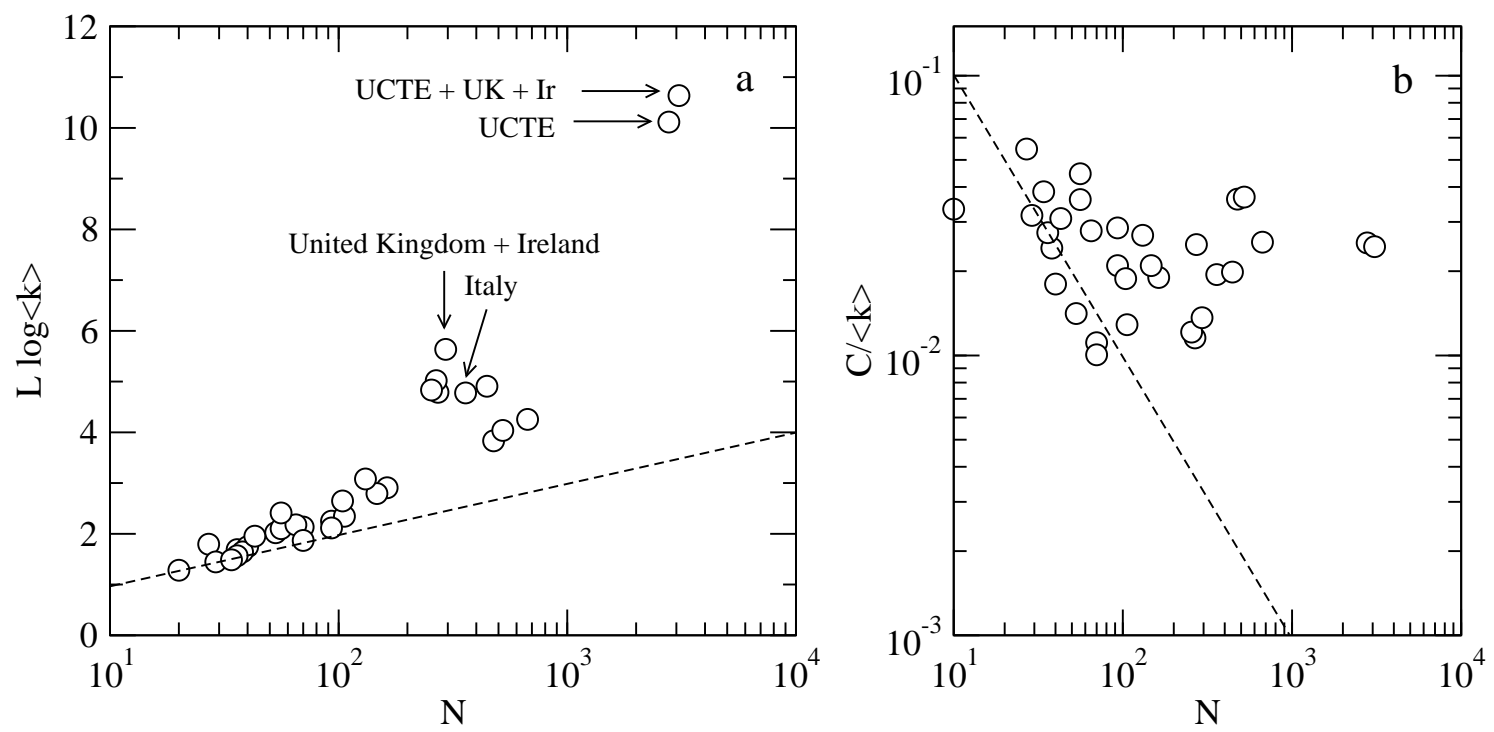

Fig. 5 Small world patterns: distance (a) and clustering (b) for the power grids investigated. Real distance is corrected by a factor of $\log <\mathrm{k}>$ and clustering by $<\mathrm{k}>$. Dashed lines signal the expected values for random graphs. It can be seen that larger networks involve larger deviations from the random cases.

Figure 5 shows the values of $d \log <k>$ and $C /<k>$ compared to those of $1 / N$ and $\log (N)$, respectively, for the 33 different power grids analyzed. It can be seen that $C / C_{\text {rand }}>1$ for most of the grids. Values of $C / C_{\text {rand }}$ of more than one order of magnitude are achieved by the largest 
power grids while $d / d_{\text {rand }}$ remains in the same order of magnitude for whatever size of the network. A similar pattern has been observed in electronic circuits [Ferrer i Cancho et al., 2001].

From a structural point of view, every country's grid has evolved in order to connect production sites with consumption sites within its own borders. In small countries everything is at hand and long distance connections are no needed to expand the grid. On the contrary, in big countries (and consequently, with an increasing number of nodes) long distance connections become more and more necessary when connecting production and consumption. At the top UCTE level, the need to exchange energy between countries forces long distance connections to cross borders and to connect sites never connected before.

\section{Static tolerance to errors and attacks}

The usual approach to the analysis of networks' static tolerance to errors and attacks seeks the relation between node deletion (without the need of redistributing any quantity transported by the network) and global connectivity (existence and relative size of the connected component, after such a deletion). An error simulation would be based on the random deletion of nodes while an attack simulation would be based on the deletion in decreasing order of the most connected (higher degree) ones. The experimental results for the 33 different power grid networks are shown in Fig. 6(b). Under random failure, simulations show a monotonically decrease of the relative network size of the connected component $S$ with the increasing fraction $f$ of nodes eliminated (orange circles). On the other hand, selective removal of the most connected nodes (blue dots), shows a much more dramatic size reduction of the connected component for the same fraction of nodes eliminated. This fact, in agreement with similar investigations done, for example, with ant galleries of networks [Buhl et al., 2004] and street networks of urban settlements [Buhl et al., 2006], clearly suggests different network behaviour upon different forms of static deletion of nodes.

In addition to numerical results [Albert et al., 2000; Motter \& Lai, 2002; Crucitti et al., 2003], the analytical approach to study tolerance to errors and attacks has been traditionally based in percolation theory. In this sense, the network percolates below a critical probability $f_{c}$ related to the presence or absence of a specific number of edges. Its study can be then mapped into a standard percolation problem for errors and, with few modifications, for attacks as well [Boccaletti et al., 2006]. Specifically for the static tolerance to errors, it has been shown [Molloy \& Reed, 1998] that the condition for having a giant component $S_{\infty}$ in a graph $\Omega$ is

$$
\left\langle k^{2}\right\rangle-2\langle k\rangle=\sum_{k} k(k-2) P(k)>0
$$

For randomly deleted nodes, it has been shown [Cohen et al., 2000] that the critical fraction $f_{c}$ is

$$
f_{c}=1-\frac{1}{\left(\left\langle k^{2}\right\rangle /\langle k\rangle-1\right)}
$$

Considering the exponential degree distribution of the European power grid (Eq. 1), we have $\langle k\rangle=\gamma$ and $\left\langle k^{2}\right\rangle=2 \gamma^{2}$, and thus

$$
f_{c}=1-\frac{1}{2 \gamma-1}
$$

For $\bar{\gamma} \cong 1.8$, we have a predicted value $f_{c} \approx 0.61$. The experimental values of $f_{c}$ for the random removal of nodes and for the different data sets analyzed are shown in Fig. 7. 

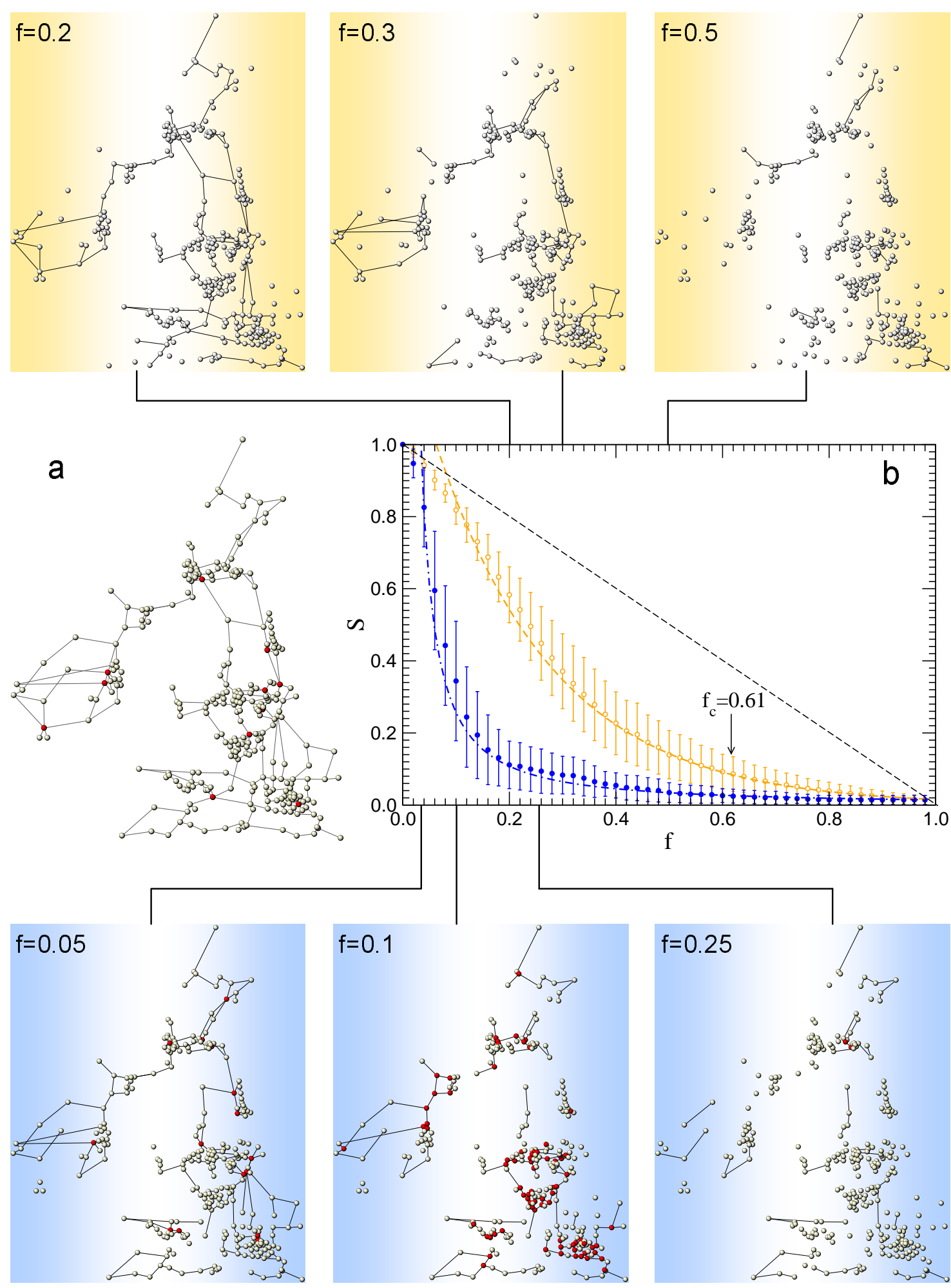

Fig. 6 Static tolerance to failures and attacks for the 33 networks analyzed (United Kingdom and Ireland power grid graph taken as an example). (a) United Kingdom and Ireland power grid original spatial graph, with the most connected nodes highlighted in red. (b) Static tolerance to random (orange) and selective (blue) removal of a fraction $f$ of nodes, measured by the relative size $S$ of the largest connected component for every network analyzed (with an analytically found critical fraction $f_{c}=0.61$ for the random case). Whiskers stand for the standard deviation. For the sample power grid (United Kingdom and Ireland), snapshot figures illustrate three random (upper orange) and three selective (lower blue) experimental results. For the United Kingdom and Ireland power grid, a progressive random removal of nodes gives a completely disconnected graph when a fraction $f_{c} \cong 0.5$ is reached, while selective removal of the most connected ones causes the grid to reach this limit sooner, for $f_{c} \cong 0.25$ (in this last case, nodes highlighted in red note those ones prone to disappear at the next time step). 
As we can see, the value of the critical fraction remains quite invariable and independent of the network size, as it should be for exponential degree distribution networks, and in completely agreement with the predicted value of $f_{c}$. An equivalent study for the case of static tolerance to attacks will be presented elsewhere.

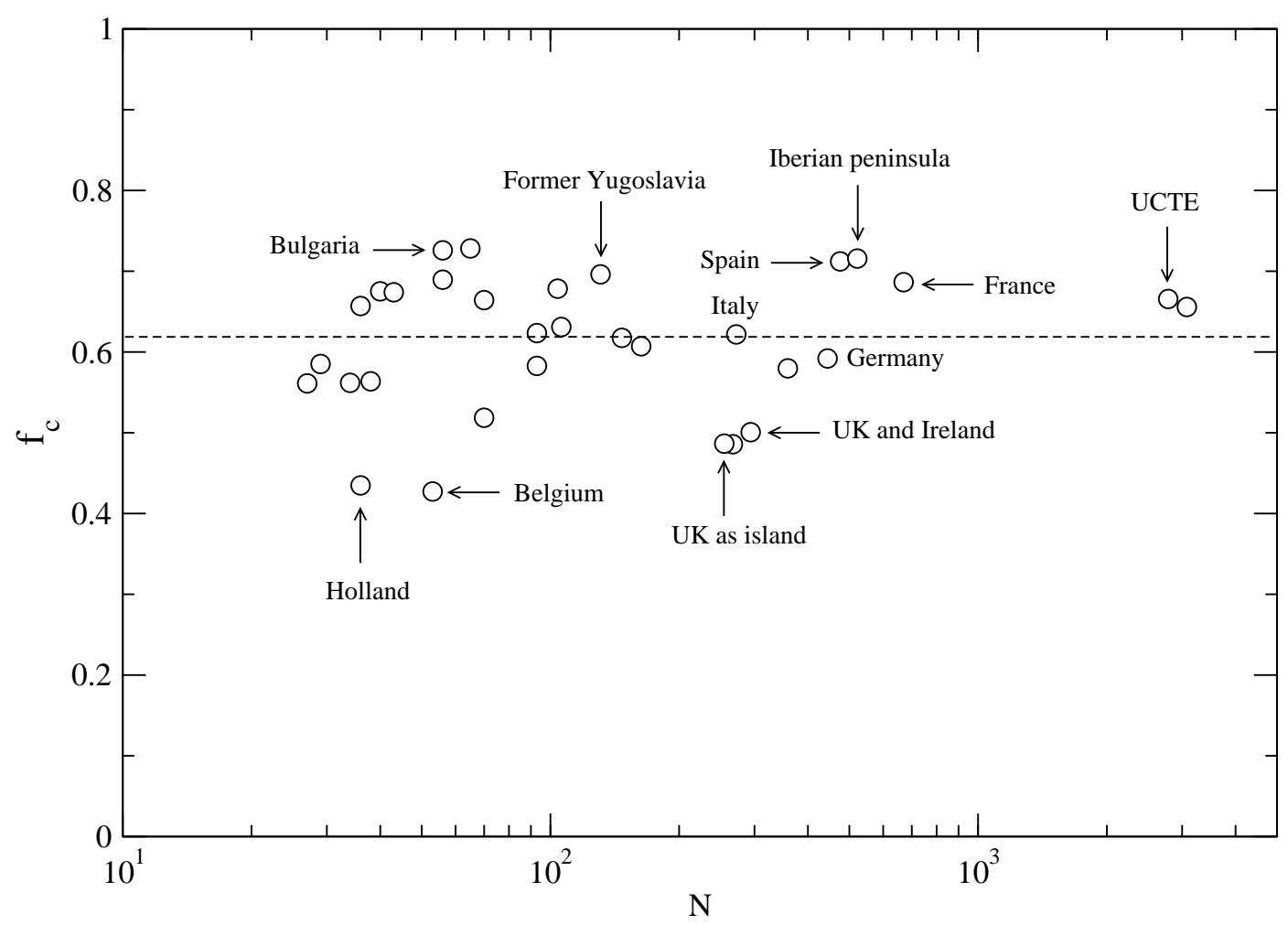

Fig. 7 Experimental values for the random removal critical fraction $f_{c}$ for every data set analyzed, as a function of the network size $N$. All values move around the predicted critical fraction $f_{c}=1-[1 /(2 \gamma-1)]$ (dashed line).

As it has been stated previously, the degree distributions of the different European power grids analyzed are exponential. That means that they are not like the highly skewed scale-free distributions typically found in other complex networks. In scale free networks, the degree distribution follows a power law, where a very few nodes have many connections and most nodes have few connections. Instead, planar networks in general, and the European power grid in particular, display less skewed exponential or uniform degree distributions.

Networks with highly skewed link distribution characterized by power laws appear very sensitive to losing those highly connected nodes (or $h u b s$ ), while relatively robust to randomly losing the more highly abundant, less connected ones. In contrast, random networks with Poisson degree distribution, which are relatively unskewed, since nodes have similar number of connections, like power grids, should display similar responses to random and selective removal of nodes [Albert et al., 2000]. The results presented insofar suggest, as it has been done for food webs [Solé \& Montoya, 2001; Dunne et al., 2002], that networks with exponential degree distributions would be, in fact, sensitive to different types of static node removals, more alike to scale-free networks than random or gaussian ones. In a nutshell: exponential, but not that much. In spite of this, these behaviours (Fig. 6, b) seem to correlate well with an exponential function of the general form

$$
S=\alpha \exp (-\beta f)
$$


where $S$ is the relative size of the connected component and $f$ is the fraction of nodes removed. ${ }^{2}$

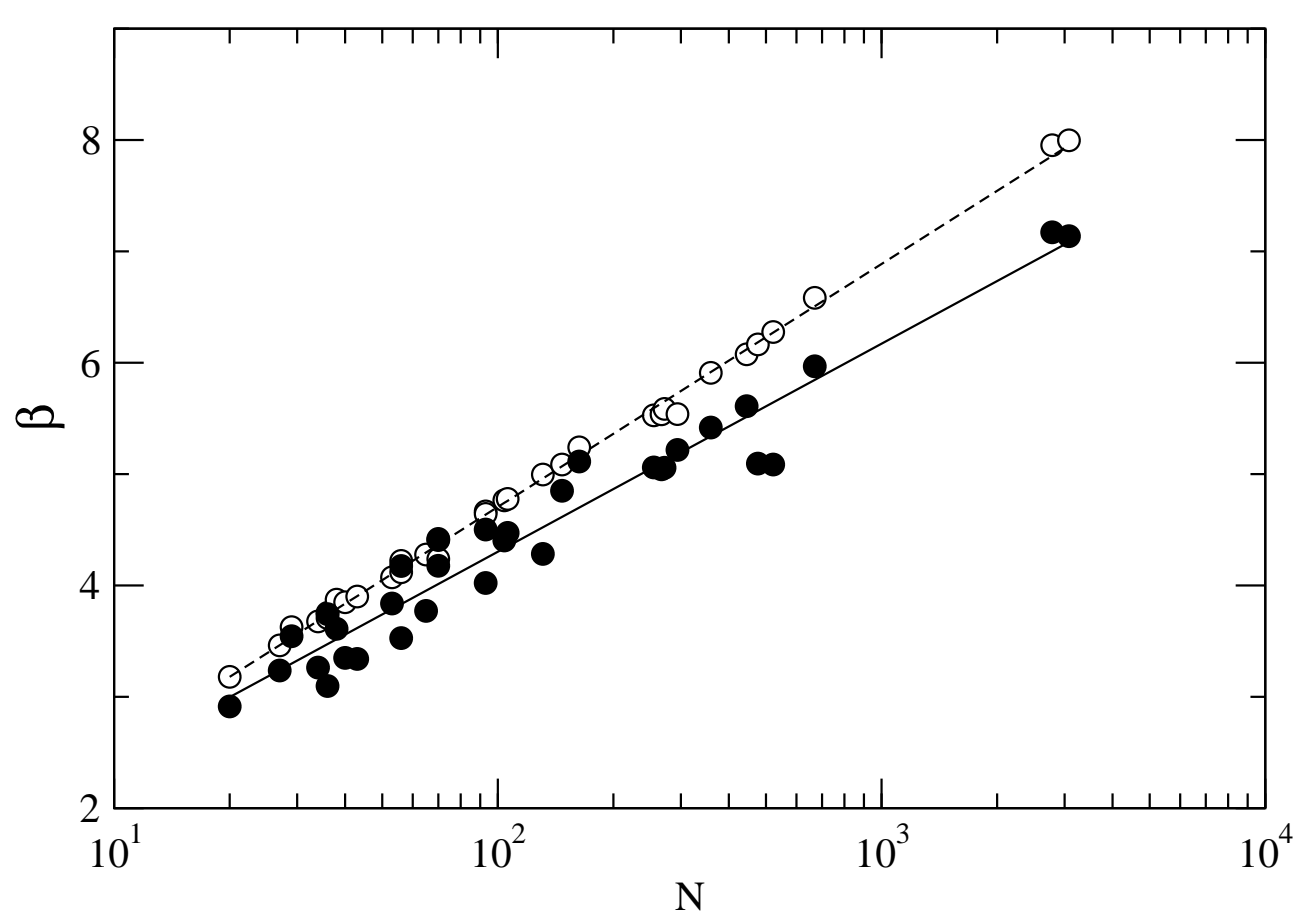

Fig. 8 Static tolerance to random (open circles) and selective (filled circles) removal of nodes, plotted as a function of the network size $N$ and the $\beta$ value of the exponential functions that correlate those behaviours from Fig. 4 . For the selective case (filled circles), the subtle though obvious dispersion from the linear fitting could stand for its different behaviour than that of a random removal, observed in Fig. 6,b.

The relation between $\beta$ and the size of the networks has been plotted in log - linear axes, in Fig. 8. As we can see, as the size of the network increases, the value of the $\beta$ exponent that better fits Eq. (6), increases at the same time. Quite intuitively, as more and more elements are introduced in the network, more prone is the system to failures, whether they come from selective or random removal, and its fragility increases as well. The more counterintuitive result arises from the fact that the increase in the value of $\beta$ is logarithmic with the size of the network. In the case of random failure, the results are very well correlated $\left(r^{2}=0.99\right)$ by the logarithmic function $\beta_{r}=\alpha_{r} \ln (N)+\theta_{r}$, with $\alpha_{r}=0.95$ and $\theta_{r}=0.34$, where $\beta$ is the exponent of the exponential function that fits the results of Fig. 6(b) and $N$ is the size of the network. The results in the case of selective attack, though offer a different observed response to deletion of nodes than that of a random removal, are also very well correlated $\left(r^{2}=0.95\right)$ by the function $\beta_{s}=\alpha_{s} \ln (N)+\theta_{s}$, with $\alpha_{s}=0.81$ and $\theta_{s}=0.56$.

\section{Discussion}

The robustness of real-world networks to the random loss of nodes ('errors') and its fragility to the selective loss of the most connected ones ('attacks') has been attributed to extremely skewed power-law distributions of links found in many small-world networks [Albert et al., 2000]. Our study shows that these responses are not unique to small-world, scale-free networks. Every single power grid studied, which have less skewed exponential degree distributions and often lack typical small-world topology, display similar patterns of response to node loss. Moreover, the difference to network response to errors and attacks appears related only to network size and

\footnotetext{
${ }^{2}$ The higher the value of $\beta$, the less skewed the function is.
} 
not to other topological measures of network complexity such as mean degree or betweenness centrality, for example (data not shown).

The evolution of both, the static tolerance to random and selective removal of nodes, plotted as a function of the network size and the exponent value of the exponential functions that correlate their node removal behaviour (Fig. 4), shows two immediate facts worth to notice: fragility increases with the size of the network and it is clearly logarithmic. We might think, rather intuitively, that the more elements are present in a system, the higher the probability that it fails. But as far as we observe the relation between the relative size $S$ of the largest connected component and the fraction $f$ of nodes deleted, the results of these static simulations should exhibit similar behaviours, quite independent of the size of the networks. On the other hand, if we consider that the increase in size of the networks is a sign of spatial or temporal evolutions, the logarithmic behaviour of the fragility with size would suggest that, though size favours fragility, evolution can, relatively, reduce it.

Recent newsworthy wide-area electrical blackouts and failures have raised many questions about the specifics of such events and the vulnerability of interconnected power systems. With the ever-growing demand for power and reliability, actual planning strategies to increase transmission systems lack basic information about the grid's complexity. One possible way to prevent propagation of disturbances is to design the system to allow for intentional separation into stable islands or interrupt small amounts of load [Madani \& Novosel, 2005]. If grid's resilience to attacks and failures is somehow related to its size and dimensions, an accurate power grid reliability analysis would have to take into account its relative increase in vulnerability in order to finally give a minimal definition of this stable island. From a spatial point of view, the definition of a geographical stable island would facilitate and improve the treatment of several different aspects related to power grid design and functioning, ranging from deregulation to spatial load forecasting and maintenance.

\section{Acknowledgements}

The authors thank the members of the Complex System Lab for useful discussions. This work has been supported by McvT grant FIS2004-05422 and by the Santa Fe Institute.

\section{References}

Albert, R., I. Albert, et al. [2004]. "Structural Vulnerability of the North American Power Grid," Physical Review E 69(025103(R)).

Albert, R., H. Jeong, et al. [2000]. "Error and attack tolerance of complex networks," Nature 406: 378-382.

Amaral, L. A. N., A. Scala, et al. [2000]. "Classes of small-world networks," Proc. Natl. Acad. Sci 97(21): 11149-11152.

Barabási, A.-L. and R. Albert [1999]. "Emergence of Scaling in Random Networks," Science 286: 509-512.

Boccaletti, S., V. Latora, et al. [2006]. "Complex networks: Structure and dynamics," Physics Reports 424: 175-308.

Buhl, J., J. Gautrais, et al. [2006]. "Topological patterns in street networks of self-organized urban settlements," European Physical Journal B 49: 513-522.

Buhl, J., J. Gautrais, et al. [2004]. "Efficiency and robustness in ant network galleries," European Physical Journal B(42): 123 -129.

Cohen, R., K. Erez, et al. [2000]. "Resilience of the Internet to Random Breakdowns," Physics Review Letters 85: 4626-4628.

Crucitti, P., V. Latora, et al. [2005]. "Locating critical lines in high - voltage electrical power grids," Fluctuation and Noise Letters 5(2): L201-L208. 
Crucitti, P., V. Latora, et al. [2003]. "Efficiency of scale-free networks: error and attack tolerance," Physica A 320: 622-642.

Dunne, J. A., R. J. Williams, et al. [2002]. "Network structure and biodiversity loss in food webs: robustness increase with connectance," Ecology Letters 5: 558-567.

Ferrer i Cancho, R., C. Janssen, et al. [2001]. "Topology of technology graphs: Small world patterns in electronic circuits," Physical Review E 64(046119).

Gastner, M. T. and M. E. J. Newman [2004]. "The spatial structure of networks," arxiv:condmat/0407680 vl.

Kinney, R., P. Crucitti, et al. [2005]. "Modeling cascading failures in the North American power grid," European Physical Journal B 46: 101-107.

Madani, S. and D. Novosel [2005]. "Taming the Power Grid," IEEE Spectrum Online(January).

Molloy, M. and B. Reed [1998]. "The Size of the Largest Component of a Random Graph on a Fixed Degree Sequence," Combinatorics, Probability and Computing 7: 295-306.

Moreno, Y., J. B. Gómez, et al. [2002]. "Instability of scale-free networks under node-breaking avalanches," Europhysics Letters 58(4): 630.

Motter, A. E. and Y.-C. Lai [2002]. "Cascade-based attacks on complex networks," Physical Review E 66(065102(R)).

Pastor Satorras, R., A. Vázquez, et al. [2001]. "Dynamical and Correlation Properties of the Internet," Physical Review Letters 87(25): 258701(4).

Pastor Satorras, R. and A. Vespignani [2004]. Evolution and Structure of Internet: A Statistical Physics Approach. Cambridge, Cambridge University Press.

Solé, R. V. and J. M. Montoya [2001]. "Complexity and fragility in ecological networks," Proc. R. Soc. London B 268: 2039-2045.

Standage, T. [1998]. The Victorian Internet. New York, Walker \& Co.

UCTE (2004). FINAL REPORT of the Investigation Committee on the 28 September 2003 Blackout in Italy, Available on-line at www.ucte.org.

UCTE [September, 2003]. "Major blackouts in Europe and North-America raise fundamental questions about the reliability of transmission systems," Newsgrid. Quarterly Bulletin of Information From UCTE(5).

Venkatasubramanian, M. V. (2003). Analyzing Blackout Events: Experience from Major Western Blackouts in 1996. Washington, Power Systems Engineering Research Center, Washington State University.

Watts, D. J. [1999]. Small Worlds. The Dynamics of Networks between Order and Randomness. Princeton, NJ, Princeton University Press.

Watts, D. J. [2003]. Six Degrees. The Science of a Connected Age. New York, W.W.Norton \& Company, Inc.

Watts, D. J. and S. H. Strogatz (1998). Collective dynamics of 'small-world' networks. Nature. 393: 440-442.

Willis, H. L. [2004]. Power Distribution Planning Reference Book, Marcel Dekker. 